\title{
Autocorrelação de um Estimador da Razão Sinal-Ruído para Adaptação de Modulação
}

\author{
Stefan Bernat Junior, Ernesto Leite Pinto e Juraci Ferreira Galdino
}

\begin{abstract}
Resumo-Em diversos sistemas de modulação adaptativa procura-se adaptar o esquema de modulação às variações do canal de propagação, com o objetivo de aumentar a eficiência espectral e manter a taxa de erro de bit abaixo de um valor máximo aceitável. A adaptação da modulação em geral é feita a cada bloco de dados transmitido, com base na estimativa de um parâmetro indicador do estado do canal. Um parâmetro útil para emprego em canais com desvanecimento plano e variante no tempo é a razão sinal-ruído por bloco, calculada com base na recepção de um bloco de dados anterior. Embora ainda pouco explorada em outros trabalhos, a autocorrelação deste parâmetro é de fundamental importância para a escolha de parâmetros do sistema de adaptação. Neste artigo, a expressão desta autocorrelação é obtida e validada por resultados de simulação de Monte Carlo, considerando-se diferentes condições de transmissão. Além disso, são apresentados resultados preliminares de aplicação da análise aqui apresentada no projeto e na otimização de parâmetros de sistemas de modulação adaptativa.
\end{abstract}

Palavras-Chave-Modulação adaptativa, eficiência espectral, desvanecimento plano.

Abstract-Several systems of adaptative modulation aim to adapt the modulation technique to the channel variations in order to improve the spectral efficienty and keep the bit error rate below a maximum acceptable value. The adaptation is usually performed by blocks of data, using estimates of a parameter related to the channel conditions. A convenient parameter for use in time-varying and flat-fading conditions is the signal-to-noise ratio per block, calculated on the basis of previously received data. Despite of not having been exploited in other works, the autocorrelation of this parameter is of utmost importance for an optimized design of the adaptation system. An analysis of this autocorrelation is here presented and validated by comparisons with Monte Carlo simulations, considering different transmission scenarios. Besides, some preliminary examples of the application of the analytical results here derived in the design and parameter optimization of adaptive modulation systems are also given.

Keywords-Adaptive modulation, spectral efficiency, flat fading.

\section{INTRODUÇÃO}

A crescente demanda por serviços multimídia que oferecem transmissão de dados, imagens, vídeo, e acesso à world wide $w e b(w w w)$, tem exigido uma ocupação do espectro cada vez mais eficiente e flexível em ambientes de comunicação que envolvem severa limitação de banda e diversos distúrbios, tais como, ruído e desvanecimento.

Com o objetivo de atender o grande aumento de demanda de serviços em sistemas de comunicações sem fio, diversas técnicas que propiciam elevada eficiência espectral vêm sendo

Stefan Bernat Junior, Ernesto Leite Pinto e Juraci Ferreira Galdino, Departamento de Engenharia Eletrica, Instituto Militar de Engenharia, Rio de Janeiro, Brasil, E-mails: stefanbernat@yahoo.com.br, ernesto@ime.eb.br, galdino@ime.eb.br. propostas. Uma dessas técnicas é a de transmissão adaptativa, que explora as condições de propagação do canal de comunicação e adapta, dinamicamente, parâmetros do sistema de transmissão como, por exemplo, esquema de modulação, taxa do código corretor de erros, e potência de transmissão [1].

Uma das técnicas de transmissão adaptativa que tem recebido grande destaque nos últimos anos é a de modulação adaptativa [2], [3], [4], [5], na qual o esquema de modulação é adaptado em resposta às condições de propagação do canal. Deste modo, procura-se melhorar a eficiência espectral quando as condições de recepção são boas ao mesmo tempo em que se atende aos requisitos mínimos da taxa de erro.

A escolha do esquema de modulação é feita com base em um parâmetro que deve apresentar boa correlação com a quantidade de erros ocorridos no bloco. Ele pode ser associado ao estado do canal de comunicação ou ao próprio desempenho do sistema, sendo de fundamental importância para o sucesso da técnica de modulação adaptativa. $\mathrm{O}$ valor deste parâmetro é estimado no receptor e enviado, através do canal de retorno, ao transmissor.

Entretanto, nos sistemas que apresentam grande retardo de ida e volta (RTT - do termo em inglês Round Trip Time), ou que empregam estrutura de bloco de dados com quantidade elevada de símbolos, ou que adotam canais cuja resposta ao impulso varia muito rapidamente, as condições de propagação podem mudar significativamente durante a transmissão de um bloco. Nestas situações, pode-se observar uma perda significativa de correlação do parâmetro de adaptação no intervalo compreendido entre a sua estimação no receptor e seu emprego no transmissor. Em conseqüência, o tipo de modulação escolhido pode não ser mais o apropriado, degradando o desempenho da técnica de modulação adaptativa. Assim sendo, este artigo propõe o uso da função de autocorrelação do parâmetro de adaptação como figura de mérito na avaliação do emprego de um sistema de modulação adaptativa.

Em canais caracterizados pelo efeito de desvanecimento plano e variante no tempo, um parâmetro que pode ser considerado para realizar a adaptação da modulação é a razão sinalruído (RSR) média por bloco na entrada do receptor, tendo em vista que, neste modelo de canal, todas as componentes de freqüência do sinal são atenuadas pelo mesmo fator.

Este artigo apresenta a expressão da autocorrelação de um estimador da razão sinal-ruído (ERSR) média por bloco em função de parâmetros típicos de um sistema de comunicações. A referida expressão é, então, validada através de simulação computacional de Monte Carlo e empregada, em conjunto com as curvas de taxa de erro e de eficiência espectral, na análise de desempenho de um sistema de modulação adaptativa.

Uma breve descrição da técnica de modulação adaptativa 
é apresentada na Seção II. A expressão da autocorrelação do ERSR é obtida na Seção III, e sua validação, através de simulação computacional, é discutida na Seção IV. Por fim, na Seção V, são sumarizadas as conclusões.

\section{A TÉCNICA DE MODULAÇÃO ADAPTATIVA}

Nos cenários em que o canal de comunicação é variante no tempo, os sistemas de comunicação que empregam técnicas de modulação fixa, fazem uso inadequado de valiosos recursos do sistema de transmissão como, por exemplo, a largura de banda, uma vez que os parâmetros do sistema de transmissão são dimensionados com base nas condições de propagação severas do canal.

Nas técnicas de modulação adaptativa, em contraposição às técnicas de modulação fixa, o esquema de modulação empregado varia ao longo da transmissão de acordo com as condições do canal. Quando o canal apresenta excelentes condições de propagação, é possível atingir taxas de erro usando esquemas de modulação com elevada eficiência espectral. Por outro lado, quando o canal apresenta severas condições de propagação, a manutenção da taxa de erro de bit (BER - do termo em inglês Bit Error Rate) requer o uso de esquemas de modulação com baixa eficiência espectral.

Logo, admitindo-se que o sistema de comunicação deva operar com uma probabilidade de erro alvo $\left(P_{\text {alvo }}\right)$, o esquema de modulação adaptativa, com base no parâmetro responsável pela adaptação, escolhe a técnica de maior eficiência espectral que garanta uma taxa de erro menor ou igual à $P_{a l v o}$.

O diagrama de blocos em banda básica de um enlace de comunicação digital que emprega a técnica de modulação adaptativa é ilustrado na Figura 1.

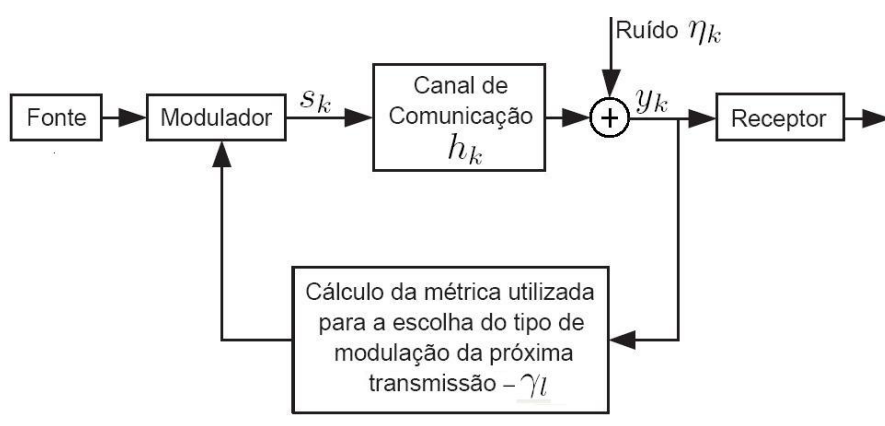

Fig. 1. Diagrama básico de um enlace de comunicação que emprega a técnica de modulação adaptativa.

Assume-se que a fonte de informação gera bits estatisticamente independentes e equiprováveis, os quais são entregues ao modulador digital para fins de mapeamento em símbolos $s_{k}$, de energia média $\sigma_{s}^{2}$, dentre os disponíveis no alfabeto do esquema de modulação. Em geral, as técnicas de modulação adaptativa empregam esquemas de modulação M-QAM, do termo em inglês Multilevel Quadrature Amplitude Modulation, em razão do bom compromisso entre eficiência espectral e taxa de erro de bit que esses esquemas oferecem [6].

Considerando canais de comunicação caracterizados pelo efeito de desvanecimento plano e variante no tempo, a observação na entrada do receptor é dada por:

$$
y_{k}=h_{k} s_{k}+\eta_{k}
$$

sendo $h_{k}$ o coeficiente do canal que é modelado por um processo estacionário em sentido amplo, cuja densidade espectral de potência, espalhamento Doppler, é dada pelo espectro de Jakes [7], com máximo desvio Doppler denotado por $f_{D} ; \eta_{k}$ é o ruído aditivo modelado por um processo gaussiano branco complexo de média nula e variância $\sigma_{\eta}^{2}$; e $k$ representa o índice do instante de tempo considerado.

O sinal recebido é empregado para detectar a informação transmitida, e para estimar o parâmetro $\gamma_{l}$ utilizado na escolha do esquema de modulação a ser adotado na transmissão do próximo bloco de dados. Para tal, o valor desse parâmetro é enviado, através do canal de retorno, ao transmissor.

Um estimador do parâmetro RSR média por bloco, que pode ser empregado na escolha do esquema de modulação em sistemas adaptativos, é dado por:

$$
\gamma_{l}=\frac{1}{N \hat{\sigma}_{\eta}^{2}} \sum_{k \in B_{l}}\left|y_{k}\right|^{2}-1
$$

onde $N$ é a quantidade de símbolos em um bloco de dados, $B_{l}$ é o l-ésimo bloco transmitido, e $\hat{\sigma}_{\eta}^{2}$ é uma estimativa da variância do ruído obtida durante a fase de treinamento.

Vários fatores são de fundamental importância para um bom desempenho da técnica de modulação adaptativa, como, por exemplo, o tamanho do bloco de dados, o conjunto dos esquemas de modulação e constelações adotadas, as características de desempenho do canal de retorno e seu retardo [4], o parâmetro usado para fazer a adaptação do sistema, e a sua autocorrelação.

No caso do tamanho do bloco de dados, do desvio Doppler normalizado $\left(f_{D} T\right)$, sendo $T$ a duração do símbolo, ou do RTT ser muito grande, o canal de comunicação pode mudar significativamente durante a transmissão de um bloco. Assim sendo, o tipo de modulação escolhido para o próximo bloco poderá não ser o mais apropriado, degradando o desempenho ou, até mesmo, inviabilizando o uso da técnica de modulação adaptativa. Se, por outro lado, o comprimento do bloco for muito pequeno, o fluxo de informação no canal de retorno aumenta, tornando o sistema de comunicação ineficiente. Portanto, a autocorrelação do parâmetro utilizado na escolha do esquema de modulação é de fundamental importância para análise de desempenho do emprego de um sistema de modulação adaptativa, bem como para otimizar parâmetros do sistema de transmissão como, por exemplo, o tamanho do bloco de dados.

\section{AutOCORRELAÇÃO DO ESTIMADOR DA RAZÃO SINAL-RUÍDO (ERSR)}

Tendo em vista a importância da correlação da métrica empregada na técnica de modulação adaptativa, nesta seção será obtida a expressão da autocorrelação do ERSR.

A autocorrelação do ERSR entre blocos de dados separados de $m$ blocos, com $m \neq 0$, é dada por: 
e que $R_{h}(l)=R_{h}^{*}(-l)$, temos:

$$
\begin{aligned}
& R_{\gamma}(m)= \\
& E\left[\left(\frac{1}{N \hat{\sigma}_{\eta}^{2}} \sum_{k \in B_{l}}\left|y_{k}\right|^{2}-1\right)\left(\frac{1}{N \hat{\sigma}_{\eta}^{2}} \sum_{j \in B_{l+m}}\left|y_{k}\right|^{2}-1\right)\right] \\
& =\frac{1}{N^{2}} E\left[\frac{1}{\hat{\sigma}_{\eta}^{4}}\right] \sum_{k \in B_{l}} \sum_{j \in B_{l+m}} E\left[\left|y_{k}\right|^{2}\left|y_{j}\right|^{2}\right]- \\
& \frac{2}{N} E\left[\frac{1}{\hat{\sigma}_{\eta}^{2}}\right] \sum_{k \in B_{l}} E\left[\left|y_{k}\right|^{2}\right]+1
\end{aligned}
$$

$\mathrm{Na}$ expressão acima, admitiu-se independência estatística entre $\hat{\sigma}_{\eta}^{2}$ e as observações consideradas no cálculo da métrica de adaptação. Isso é razoável considerando-se que essa estimativa é obtida durante períodos de treinamento.

Admitindo-se que $\hat{\sigma}_{\eta}^{2}$ seja obtida por um estimador despolarizado, tem-se que $\hat{\sigma}_{\eta}^{2}=\sigma_{\eta}^{2}+e_{\hat{\sigma}_{\eta}^{2}}$, na qual $e_{\hat{\sigma}_{\eta}^{2}}$ é uma variável aleatória de média nula e variância $\operatorname{Var}\left[\hat{\sigma}_{\eta}^{2}\right]$.

Empregando aproximação por série de Taylor para $\frac{1}{\hat{\sigma}_{\eta}^{2}}$, temos que:

$$
\begin{aligned}
E\left[\frac{1}{\hat{\sigma}_{\eta}^{2}}\right] & =E\left[\frac{1}{\sigma_{\eta}^{2}+e_{\hat{\sigma}_{\eta}^{2}}}\right] \\
& \cong E\left[\frac{1}{\sigma_{\eta}^{2}}+\frac{1}{\sigma_{\eta}^{4}} e_{\hat{\sigma}_{\eta}^{2}}+\frac{1}{\sigma_{\eta}^{6}} e_{\hat{\sigma}_{\eta}^{2}}^{2}\right] \\
& \cong \frac{1}{\sigma_{\eta}^{2}}\left[1+\frac{\operatorname{Var}\left(\hat{\sigma}_{\eta}^{2}\right)}{\sigma_{\eta}^{4}}\right]
\end{aligned}
$$

De modo análogo, para $\frac{1}{\hat{\sigma}_{\eta}^{4}}$, temos:

$$
E\left[\frac{1}{\hat{\sigma}_{\eta}^{4}}\right] \cong \frac{1}{\sigma_{\eta}^{4}}\left[1+3 \frac{\operatorname{Var}\left(\hat{\sigma}_{\eta}^{2}\right)}{\sigma_{\eta}^{4}}\right]
$$

Fazendo $\left[1+\frac{\operatorname{Var}\left(\hat{\sigma}_{\eta}^{2}\right)}{\sigma_{\eta}^{4}}\right]=k_{1} \mathrm{e}\left[1+3 \frac{\operatorname{Var}\left(\hat{\sigma}_{\eta}^{2}\right)}{\sigma_{\eta}^{4}}\right]=k_{2}$, e substituindo (1), (4) e (5) em (3), temos:

$$
\begin{aligned}
& R_{\gamma}(m)= \\
& \quad \frac{k_{2}}{N^{2} \sigma_{\eta}^{4}} \sum_{k \in B_{l}} \sum_{j \in B_{l+m}} E\left[\left|h_{k} s_{k}+\eta_{k}\right|^{2}\left|h_{j} s_{j}+\eta_{j}\right|^{2}\right]- \\
& \frac{2 k_{1}}{N \sigma_{\eta}^{2}} \sum_{k \in B_{l}} E\left[\left|h_{k} s_{k}+\eta_{k}\right|^{2}\right]+1
\end{aligned}
$$

Mas, pode-se mostrar que

$$
E\left[\left|h_{k} s_{k}+\eta_{k}\right|^{2}\right]=R_{h}(0) \sigma_{s}^{2}+\sigma_{\eta}^{2}
$$

e

$$
\begin{aligned}
& E\left[\left|h_{k} s_{k}+\eta_{k}\right|^{2}\left|h_{j} s_{j}+\eta_{j}\right|^{2}\right]= \\
& \quad E\left[\left|h_{k}\right|^{2}\left|h_{j}\right|^{2}\right] \sigma_{s}^{4}+2 R_{h}(0) \sigma_{s}^{2} \sigma_{\eta}^{2}+\sigma_{\eta}^{4}
\end{aligned}
$$

Dada a propriedade do momento de quarta ordem [8],

$E\left[u_{1}^{*} u_{2}^{*} u_{3} u_{4}\right]=E\left[u_{1}^{*} u_{3}\right] E\left[u_{2}^{*} u_{4}\right]+E\left[u_{2}^{*} u_{3}\right] E\left[u_{1}^{*} u_{4}\right]$

$$
E\left[\left|h_{k}\right|^{2}\left|h_{j}\right|^{2}\right]=R_{h}^{2}(0)+R_{h}^{2}(j-k)
$$

Substituindo (9) em (8), chega-se a:

$$
\begin{aligned}
& E\left[\left|h_{k} s_{k}+\eta_{k}\right|^{2}\left|h_{j} s_{j}+\eta_{j}\right|^{2}\right]= \\
& \quad\left(R_{h}^{2}(0)+R_{h}^{2}(j-k)\right) \sigma_{s}^{4}+2 R_{h}(0) \sigma_{s}^{2} \sigma_{\eta}^{2}+\sigma_{\eta}^{4}
\end{aligned}
$$

Substituindo (7) e (10) em (6), pode-se mostrar que

$$
\begin{aligned}
& R_{\gamma}(m)= \\
& \quad k_{2} \frac{\sigma_{s}^{4}}{\sigma_{\eta}^{4}} R_{h}^{2}(0)+2\left(k_{2}-k_{1}\right) \frac{\sigma_{s}^{2}}{\sigma_{\eta}^{2}} R_{h}(0)+k_{2}-2 k_{1}+1+ \\
& k_{2} \frac{\sigma_{s}^{4}}{N^{2} \sigma_{\eta}^{4}} \sum_{k \in B_{l}} \sum_{j \in B_{l+m}} R_{h}^{2}(j-k)
\end{aligned}
$$

Fazendo as seguintes mudanças de variável:

$$
\begin{aligned}
k & =\lambda+(l-1) N \\
j & =\tau+(l+m-1) N
\end{aligned}
$$

tem-se que:

$$
\sum_{k \in B_{l}} \sum_{j \in B_{l+m}} R_{h}^{2}(j-k)=\sum_{\lambda=0}^{N-1} \sum_{\tau=0}^{N-1} R_{h}^{2}(\tau-\lambda+N m)
$$

Além disso, fazendo $\beta=\tau-\lambda$ em (12) e substituindo-se o resultado em (11), chega-se a:

$$
\begin{aligned}
& R_{\gamma}(m)= \\
& \quad k_{2} \frac{\sigma_{s}^{4}}{\sigma_{\eta}^{4}} R_{h}^{2}(0)+2\left(k_{2}-k_{1}\right) \frac{\sigma_{s}^{2}}{\sigma_{\eta}^{2}} R_{h}(0)+k_{2}-2 k_{1}+1+ \\
& k_{2} \frac{\sigma_{s}^{4}}{N^{2} \sigma_{\eta}^{4}} \sum_{\beta=-N+1}^{N-1}(N-|\beta|) R_{h}^{2}(\beta+N m)
\end{aligned}
$$

onde $m \neq 0$ (blocos diferentes).

Para o mesmo bloco de dados, a autocorrelação será dada por:

$$
\begin{aligned}
R_{\gamma}(0)= & E\left[\left(\frac{\frac{1}{N} \sum_{k \in B_{l}}\left|y_{k}\right|^{2}}{\hat{\sigma}_{\eta}^{2}}-1\right)\left(\frac{\frac{1}{N} \sum_{j \in B_{l}}\left|y_{j}\right|^{2}}{\hat{\sigma}_{\eta}^{2}}-1\right)\right] \\
= & \frac{k_{2}}{N^{2} \sigma_{\eta}^{4}} \sum_{k \in B_{l}} E\left[\left|h_{k} s_{k}+\eta_{k}\right|^{4}\right]+ \\
& \frac{k_{2}}{N^{2} \sigma_{\eta}^{4}} \sum_{k \in B_{l}} \sum_{j \in B_{l}, j \neq k} E\left[\left|h_{k} s_{k}+\eta_{k}\right|^{2}\left|h_{j} s_{j}+\eta_{j}\right|^{2}\right]- \\
& \frac{2 k_{1}}{N \sigma_{\eta}^{2}} \sum_{k \in B_{l}} E\left[\left|h_{k} s_{k}+\eta_{k}\right|^{2}\right]+1
\end{aligned}
$$

Mas, pela propriedade do momento de quarta ordem, temos: 


$$
\begin{aligned}
& E\left[\left|h_{k} s_{k}+\eta_{k}\right|^{4}\right]= \\
& 2 R_{h}^{2}(0) \sigma_{s}^{4}+4 R_{h}(0) \sigma_{s}^{2} \sigma_{\eta}^{2}+2 \sigma_{\eta}^{4}
\end{aligned}
$$

Logo, substituindo (7), (10), e (15), em (14), pode-se mostrar que:

$$
\begin{aligned}
& R_{\gamma}(0)= \\
& k_{2} \frac{(N+1)}{N} \frac{\sigma_{s}^{4}}{\sigma_{\eta}^{4}} R_{h}^{2}(0)+ \\
& 2\left[k_{2}\left(\frac{N+1}{N}\right)-k_{1}\right] \frac{\sigma_{s}^{2}}{\sigma_{\eta}^{2}} R_{h}(0)+k_{2}\left(\frac{N+1}{N}\right)- \\
& 2 k_{1}+1+k_{2} \frac{\sigma_{s}^{4}}{N^{2} \sigma_{\eta}^{4}} \sum_{k=0}^{N-1} \sum_{j=0, j \neq k}^{N-1} R_{h}^{2}(j-k)
\end{aligned}
$$

Fazendo a mudança de variável: $\beta=j-k$, temos:

$$
\begin{aligned}
& R_{\gamma}(0)= \\
& k_{2} \frac{(N+1)}{N} \frac{\sigma_{s}^{4}}{\sigma_{\eta}^{4}} R_{h}^{2}(0)+ \\
& 2\left[k_{2}\left(\frac{N+1}{N}\right)-k_{1}\right] \frac{\sigma_{s}^{2}}{\sigma_{\eta}^{2}} R_{h}(0)+k_{2}\left(\frac{N+1}{N}\right)- \\
& 2 k_{1}+1+k_{2} \frac{\sigma_{s}^{4}}{N^{2} \sigma_{\eta}^{4}} \sum_{\beta=-N+1, \beta \neq 0}^{N-1}(N-|\beta|) R_{h}^{2}(\beta)
\end{aligned}
$$

Portanto, a autocorrelação $R_{\gamma}(m)$ do ERSR média por bloco definido na Equação 2 é dada por:

- $m=0$ :

$$
\begin{aligned}
& R_{\gamma}(0)= \\
& k_{2} \frac{(N+1)}{N} \frac{\sigma_{s}^{4}}{\sigma_{\eta}^{4}} R_{h}^{2}(0)+ \\
& 2\left[k_{2}\left(\frac{N+1}{N}\right)-k_{1}\right] \frac{\sigma_{s}^{2}}{\sigma_{\eta}^{2}} R_{h}(0)+k_{2}\left(\frac{N+1}{N}\right)- \\
& 2 k_{1}+1+k_{2} \frac{\sigma_{s}^{4}}{N^{2} \sigma_{\eta}^{4}} \sum_{\beta=-N+1, \beta \neq 0}^{N-1}(N-|\beta|) R_{h}^{2}(\beta)
\end{aligned}
$$

- $m \neq 0$ :

$$
\begin{aligned}
& R_{\gamma}(m)= \\
& k_{2} \frac{\sigma_{s}^{4}}{\sigma_{\eta}^{4}} R_{h}^{2}(0)+2\left(k_{2}-k_{1}\right) \frac{\sigma_{s}^{2}}{\sigma_{\eta}^{2}} R_{h}(0)+k_{2}-2 k_{1}+ \\
& 1+k_{2} \frac{\sigma_{s}^{4}}{N^{2} \sigma_{\eta}^{4}} \sum_{\beta=-N+1}^{N-1}(N-|\beta|) R_{h}^{2}(\beta+N m)
\end{aligned}
$$

onde $k_{1}=\left[1+\frac{\operatorname{Var}\left(\hat{\sigma}_{\eta}^{2}\right)}{\sigma_{\eta}^{4}}\right]$ e $k_{2}=\left[1+3 \frac{\operatorname{Var}\left(\hat{\sigma}_{\eta}^{2}\right)}{\sigma_{\eta}^{4}}\right]$

\section{RESUltados NUMÉRICOS}

Nesta seção são apresentados resultados numéricos relativos à validação e à aplicação da análise desenvolvida na seção anterior. Para obtenção destes resultados considerou-se o conhecimento da variância do ruído no cálculo de $\gamma_{l}$. Assim sendo, $\operatorname{Var}\left(\hat{\sigma}_{\eta}^{2}\right)=0$, de modo que $k_{1}=1$ e $k_{2}=1$.

Foram consideradas diferentes condições de transmissão, caracterizadas pela razão entre a energia média do símbolo e energia média do ruído $\left(\frac{\sigma_{s}^{2}}{\sigma_{\eta}^{2}}\right)$, desvio Doppler normalizado $\left(f_{D} T\right)$, e tamanho do bloco de dados $(N)$.

As curvas empíricas da autocorrelação foram obtidas a partir da simulação de 10000 realizações independentes da transmissão de blocos de dados. Para cada uma destas transmissões foi avaliada uma estimativa parcial da autocorrelação do parâmetro de adaptação ERSR. A estimativa final foi calculada como a média destas estimativas parciais.

A Figura 2 apresenta curvas da autocorrelação do ERSR em função do índice do bloco, obtidas com a expressão analítica e via simulação, com $f_{D} T=10^{-5}, N=100$, e para $\frac{\sigma_{s}^{2}}{\sigma_{\eta}^{2}}=$ $10 d B$ e $\frac{\sigma_{s}^{2}}{\sigma_{\eta}^{2}}=20 d B$. Pode-se observar um excelente ajuste entre as curvas teóricas e as empíricas.

Comparando-se a Figura 2(a) com a Figura 2(b), verificase que, com exceção da amplitude, as curvas apresentam o mesmo comportamento para máximos e mínimos. Na Figura 2(a), para $\frac{\sigma_{s}^{2}}{\sigma_{\eta}^{2}}=10 d B$, observa-se que a autocorrelação varia entre 100 e 200. Já na Figura 2(b), para $\frac{\sigma_{s}^{2}}{\sigma_{\eta}^{2}}=20 d B$, a autocorrelação varia entre 10000 e 20000 . Isto ocorre devido ao fato de que o aumento da razão $\frac{\sigma_{s}^{2}}{\sigma_{\eta}^{2}}$ provoca um ganho na amplitude da autocorrelação.

Outra importante observação é a existência de um patamar mínimo para o valor da autocorrelação. Este nível depende exclusivamente da razão $\frac{\sigma_{s}^{2}}{\sigma_{\eta}^{2}}$.
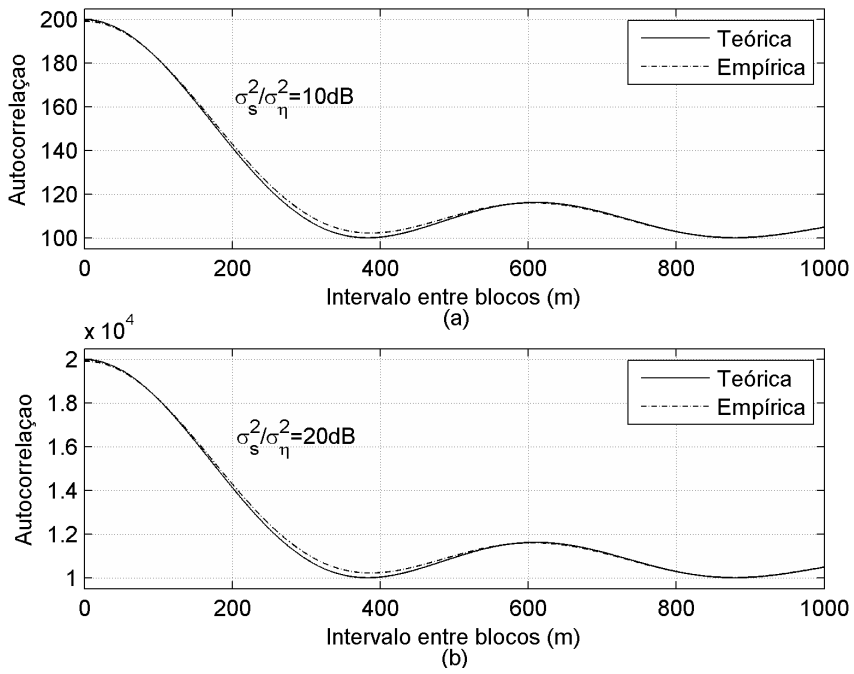

Fig. 2. Autocorrelação do ERSR em função do índice do bloco, para a expressão analítica e valores simulados, e para: $(a) \frac{\sigma_{s}^{2}}{\sigma_{\eta}^{2}}=10 d B$; e $(b) \frac{\sigma_{s}^{2}}{\sigma_{\eta}^{2}}=$ $20 d B$.

Outra comparação entre curvas analíticas e empíricas de 
autocorrelação do ERSR em função do índice do bloco é mostrada na Figura 3. Estas curvas foram obtidas com $\frac{\sigma_{s}^{2}}{\sigma_{\eta}^{2}}=$ $10 d B$ e $N=100$, e para $f_{D} T=10^{-5}$ e $f_{D} T=10^{-4}$. Observa-se novamente um ótimo ajuste entre as curvas teóricas e as empíricas. Verifica-se, também, na Figura 3 que, quanto menor é o valor do produto $f_{D} T$, maior é a correlação entre as estimativas do ERSR. Isto se deve ao fato de o canal variar mais lentamente com a redução de $f_{D} T$, aumentando, assim, a correlação entre as amostras do ERSR.

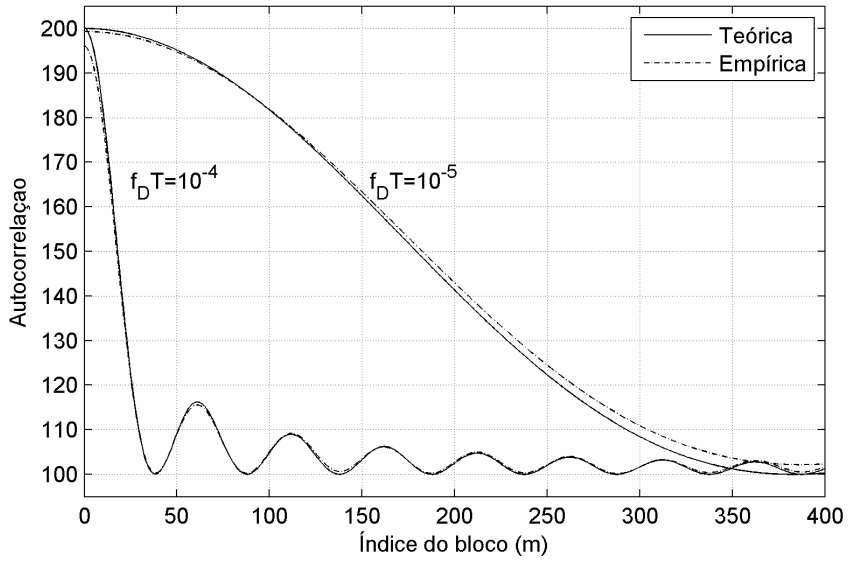

Fig. 3. Autocorrelação do ERSR em função do índice do bloco, para a expressão analítica e valores simulados, e para $f_{D} T=10^{-4}$ e $f_{D} T=$ $10^{-5}$.

A Figura 4 apresenta curvas empíricas e analíticas da autocorrelação do ERSR em função do índice do bloco, obtidas com $\frac{\sigma_{s}^{2}}{\sigma_{\eta}^{2}}=10 d B$ e $f_{D} T=10^{-5}$, e para $N=100$ e $N=200$. Pode-se notar mais uma vez um ajuste muito bom entre as curvas teóricas e as obtidas por simulação. Observase, ainda, que, aumentando a quantidade de símbolos no bloco, a correlação do ERSR diminui. Isto se deve ao fato de que quanto maior for o tamanho do bloco $(N)$, maior será o tempo necessário para transmiti-lo e, em conseqüência, menos correlacionadas serão as amostras do ERSR obtidas em cada bloco.

A fim de demonstrar a importância da autocorrelação do ERSR para o emprego da técnica de modulação adaptativa, considerou-se um sistema de comunicações que emprega o ERSR apresentado na Equação 2 e os esquemas de modulação BPSK, 4-QAM, 16-QAM e 64-QAM. A Tabela I apresenta os limiares de decisão para a escolha do esquema de modulação. Estes limiares foram fixados de modo a escolher a técnica de maior eficiência espectral que garanta uma taxa de erro menor ou igual à $P_{\text {alvo }}=10^{-2}$ [9]. Considerou-se o canal de retorno ideal, ou seja, sem erro e sem retardo. Para efeito de decisão dos símbolos, a resposta ao impulso do canal foi considerada conhecida.

Essa técnica de modulação adaptativa foi aplicada em três modelos de sistema de comunicações distintos descritos na Tabela II.

Os valores da autocorrelação $R_{\gamma}(1)$ contidos na Tabela II foram obtidos a partir da expressão analítica apresentada neste artigo para os respectivos parâmetros de cada sistema e para

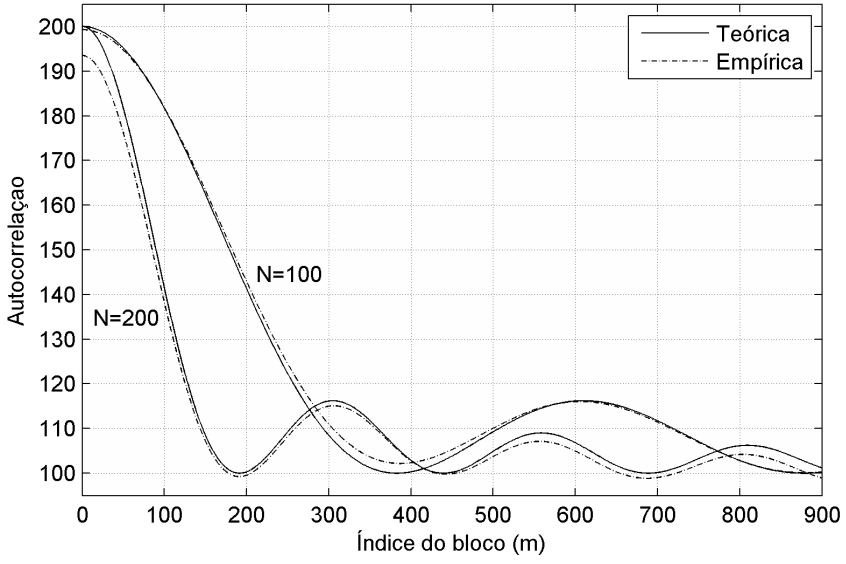

Fig. 4. Autocorrelação do ERSR em função do índice do bloco, para a expressão analítica e valores simulados, e para tamanhos de bloco $N=100$ e $N=200$

TABELA I

LIMIARES DE DECISÃO PARA ESCOLHA DO ESQUEMA DE MODULAÇÃO

\begin{tabular}{c|c}
\hline Esquema de modulação & $\gamma_{l}$ em dB \\
\hline BPSK & $\gamma_{l} \leq 12$ \\
\hline 4-QAM & $12<\gamma_{l} \leq 19$ \\
\hline 16-QAM & $19<\gamma_{l} \leq 25$ \\
\hline 64-QAM & $\gamma_{l}>25$ \\
\hline
\end{tabular}

$\frac{\sigma_{s}^{2}}{\sigma_{\eta}^{2}}=10 d B$

O valor de $R_{\gamma}(1)$ denota a correlação entre amostras do ERSR obtida em blocos de dados sucessivos. Tendo em vista que foi admitido canal de retorno ideal, a diferença entre $R_{\gamma}(1)$ e $R_{\gamma}(0)$ indica a perda de correlação ocorrida entre a estimativa do ERSR e seu uso na adaptação.

Pode-se verificar que o modelo I possui maior valor de autocorrelação que o modelo II. Uma vez que para os dois modelos são adotados os mesmos valores de $N$ e RSR, a redução da autocorrelação é determinada basicamente pelo valor de $f_{D} T$, sendo que, quanto maior for o valor de $f_{D} T$, menor será o valor de $R_{\gamma}(1)$. Assim sendo, as amostras sucessivas ERSR do modelo I são mais correlacionadas que as do modelo II. Esta diferença na autocorrelação do parâmetro de adaptação é refletida no desempenho da técnica de modulação adaptativa, como se pode verificar na Figura 5.

A fim de demonstrar que essa degradação de desempenho foi conseqüência da perda de correlação das amostras do ERSR, buscou-se, nas curvas analíticas da autocorrelação do modelo I, um valor de índice de bloco $m$ que correspondesse ao valor da autocorrelação do modelo II para $m=1$.

Verificou-se, na curva de autocorrelação do modelo I, que

TABELA II

PARÂMETROS DOS MODELOS AVALIADOS

\begin{tabular}{c|c|c|c}
\hline Sistema & Doppler $\left(f_{D} T\right)$ & Tamanho $(\mathrm{N})$ & $R_{\gamma}(1)$ \\
\hline Modelo I & $10^{-5}$ & 100 & 199,99 \\
\hline Modelo II & $10^{-3}$ & 100 & 179,76 \\
\hline Modelo III & $10^{-5}$ & 10500 & 177,97 \\
\hline
\end{tabular}




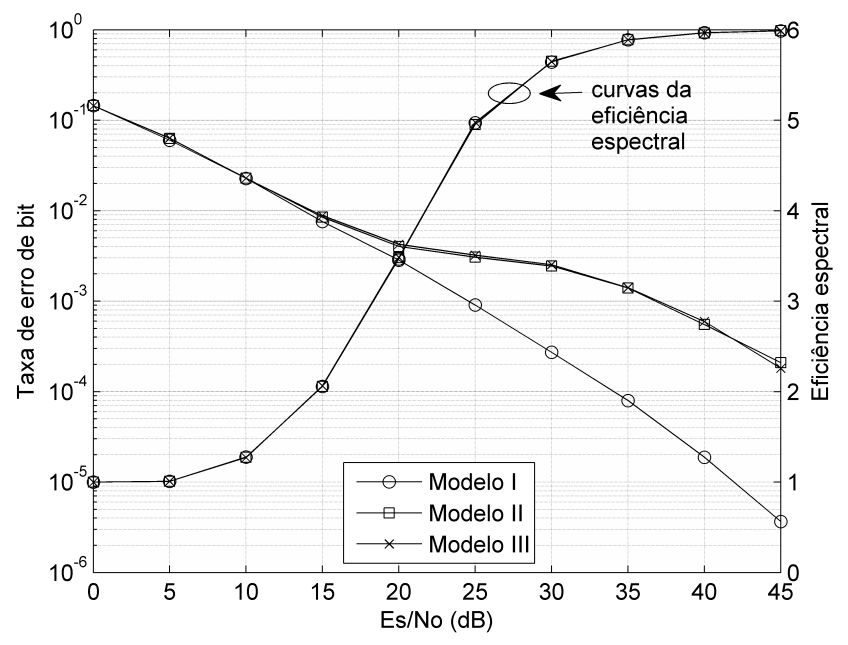

Fig. 5. Taxa de erro de bit e eficiência espectral em função de $\frac{E s}{N o}$ para um sistema de modulação adaptativa que emprega esquemas BPSK, 4-QAM, 16-QAM e 64-QAM, em canal plano com $f_{D} T=10^{-3}$ e $f_{D} T=10^{-5}$.

$R_{\gamma I}(105)=179,94$. Portanto, para que os modelos I e II tivessem valores aproximados de $R_{\gamma}(1)$, determinou-se o modelo III com a mesma $f_{D} T$ do modelo I, porém com tamanho de bloco $N=105 \times 100=10500$. Assim sendo, $R_{\gamma I I I}(1)=177,97$

Curvas de taxa de erro de bit dos três sistemas simulados são apresentadas na Figura 5. Também são mostradas curvas de eficiência espectral, as quais foram obtidas nas mesmas condições de simulação das curvas da taxa de erro. Assim sendo, para cada curva de BER, existe uma curva correspondente de eficiência espectral.

A $P_{\text {alvo }}=10^{-2}$ foi atingida em $\frac{E s}{N o}=13.8 \mathrm{~dB}$ para o modelo I e em $\frac{E s}{N_{o}}=14.3 d B$ para os modelos II e III, conforme se verifica na Figura 5. A $P_{\text {alvo }}$ somente pôde ser alcançada nesses valores de $\frac{E s}{N o}$ em virtude da técnica de modulação adaptativa implementada neste trabalho considerar o sistema sempre transmitindo, independente do valor de $\frac{E s}{N o}$. Assim sendo, até atingir a $P_{\text {alvo }}$, os modelos apresentam suas curvas de taxa de erro de bit praticamente coincidentes com a curva da taxa de erro do BPSK, o qual possui o melhor desempenho em termos desta figura de mérito em comparação com os demais esquemas aqui empregados.

Entretanto, já a partir de $\frac{E s}{N_{o}}=5 d B$ foi possível o emprego de esquemas de modulação com maior quantidade de bits por símbolo que o BPSK, conforme se verifica na Figura 5, proporcionando um aumento na eficiência espectral do sistema e demonstrando o ganho obtido no emprego da técnica de modulação adaptativa em relação à técnica de modulação fixa.

Além disso, verifica-se, ainda, que para valores de $\frac{E s}{N o}$ acima de $10 d B$, ocorre uma mesma degradação de desempenho dos modelo II e III, em relação ao modelo I. Este fato é conseqüência do valor da correlação assumido por cada modelo, de acordo com a Tabela II. Nos modelos II e III ocorre uma perda significativa de correlação do parâmetro de adaptação no intervalo compreendido entre a sua estimação no receptor e seu emprego no transmissor. Em conseqüência, o tipo de modulação escolhido para a transmissão dos blocos, em sua maioria, não foi o mais apropriado, causando uma degradação no desempenho da técnica de modulação adaptativa em termos de taxa de erro de bit. O modelo I, por sua vez, apresenta maior valor para $R_{\gamma}(1)$ e, por conseqüência, um melhor desempenho em termos desta figura de mérito.

Por fim, uma importante observação reside no fato de que o desempenho da técnica de modulação adaptativa pode ser mantido, mesmo em diferentes condições de propagação, através da análise da autocorrelação do parâmetro de adaptação. Alterando-se parâmetros do sistema de transmissão como, neste caso, o tamanho do bloco de dados de $N=100$ para $N=10500$, foi possível inferir sobre o desempenho do modelo I, de modo que se tornasse coincidente com o desempenho do modelo II, mesmo em condições de diferentes valores de $f_{D} T$.

\section{CONCLUSÕES}

Neste trabalho foi obtida a expressão analítica da função autocorrelação de um estimador da razão sinal-ruído média por bloco (ERSR) na entrada do receptor, para aplicação em sistemas de modulação adaptativa em canais com desvanecimento plano e variante no tempo.

Foram apresentados diversos resultados de validação da expressão analítica obtida, através de comparação de resultados por ela produzidos com estimativas obtidos por simulação computacional. Nesta comparação foram consideradas diferentes condições de transmissão caracterizadas pelos valores da razão entre a variância dos símbolos e a variância do ruído, pelo desvio Doppler máximo normalizado, e pelo tamanho do bloco de dados. Em todos os casos investigados observou-se um excelente ajuste entre os resultados analíticos e empíricos.

Foram ainda apresentados alguns resultados preliminares de aplicação da análise aqui apresentada no projeto e na otimização de parâmetros de sistemas com modulação adaptativa. Novas aplicações desta análise serão investigadas na continuação deste trabalho.

\section{REFERÊNCIAS}

[1] Jain, Payal and Buehrer, R. Michael. "Implementation of Adaptive Modulation on the Sunrise Software Radio". IEEE Transactions on Communicatons, 2002.

[2] Mohammad, Maruf and Buehrer, Michael. "On the Impact of SNR Estimation Error on Adaptive Modulation". IEEE Transactions on Communicatons, vol. 9, no. 6, June 2005.

[3] Conti, Andrea; Win, Moe Z. and Chiani, Marco. "Invertible Bounds for $M-Q A M$ in Rayleigh Fading". IEEE Transactions on Communicatons, vol. 4, no. 5, 2005.

[4] Ekpenyoung, Anthony E.; Huang, Yih-Fang. "Feedback-Detection Strategies for Adaptive Modulation Systems". IEEE Transactions on Communicatons, vol. 4, no. 5, 2005.

[5] Rodrigues, Alessandra. "Modulação Adaptativa para canais WSS-US baseada na detecção de erros de equalização". Dissertação de Mestrado - Instituto Militar de Engenharia (IME), 2006.

[6] Proakis, J. G. "Digital Comunications". MCGraw-Hill, 1995.

[7] Parsons, J. D. "The Mobile Radio Propagation Channel". John Wiley, 1992.

[8] Haykin, S. "Adaptive Filter Theory". Prentice-Hall, Englewood-Cliffs, 1996.

[9] Wong, C. H. and Hanzo, Lajos. "Upper-Bound Performance of a WideBand Adaptive Modem". IEEE Transactions on Communicatons, vol. 48, no. 3, March 2000. 